\title{
ESTADO NUTRICIONAL DOS PRÉ-ESCOLARES INGRESSANTES NOS CENTROS DE EDUCAÇÃO E ALIMENTAÇÃO \\ DO PRÉ-ESCOLAR
}

\author{
Maria Helena D'Aquino Benicio ** \\ Carlos Augusto Monteiro** \\ Maria José Pontieri *** \\ Yaro Ribeiro Gandra** \\ Fernão Dias de Lima ****
}

\begin{abstract}
BENiClO, M. H. D'A. et al. Estado nutricional dos pré-escolares ingressantes nos Centros de Educação e Alimentação do Pré-escolar. Rev. Saúde públ., S. Paulo, 15 (supl.) :33-9, 1981 .

RESUMO: Descreve-se o estado nutricional da população de pré-escolares ingressantes nos Centros de Educação e Alimentação do Pré-escolar (CEAPE) por intermédio de medidas antropométricas. A prevalência da desnutrição protéico calórica encontrada foi igual a $46,8 \%$ com graus moderados e graves em $5,8 \%$ dos casos, situação esta, intermediária entre a descrita em duas outras populações desfavorecidas economicamente no Estado de São Paulo, Brasil.
\end{abstract}

UNITERMOS: Pré-escolares, estado nutricional. CEAPE.

\section{INTRODUÇAO}

Trabalho anterior: explicitou a filosofia e objetivos do programa CEAPE (Centro de Educação e Alimentação do Pré-Escolar) identificando-o como alternativa de baixo custo para atendimento ao pré-escolar.

Seu baixo custo operacional permite extensão de cobertura aos segmentos populacionais mais desfavorecidos do ponto de vista social e econômico, frequientemente desassistidos pelos programas tradicionais de atendimento a esta faixa etária.

O nivel de renda das familias dos pré-escolares "Ceapenses" confirmam esta as- sertiva na medida em que cerca de $90 \%$ dos pré-escolares, pertence a famílias situadas abaixo de um salário mínimo per capita. Vários estudos $7,8,9,10$ concordam quanto an fato de que neste nivel de renda a desnutrição protéico calórica (dpc) surge como um dos problemas prioritários de saúde dada a impossibilidade de compra da quantidade suficiente de alimentos para o preenchimento das necessidades calóricas dos individuos.

O objetivo deste trabalho é estudar a prevalência da dpc nos pré-escolares ao ingressarem no Programa.

* Convênio 10/77 - INAN/DN/FSP/USP.

** Do Departamento de Nutrição da Faculdade de. Saúde Pública da USP - Av. Dr. Arnaldo, 715 - 01255 - São Paulo, SP - Brasil.

*** Do Programa CEAPE - Departamento de Nitrição da Faculdade de Saúde Pública da USP

**** Do Departamento de Epidemiologia da Faculdade de Saúde Pública da USP - Ar. Dr. Arnaldo, 715 - 01255 - Sảo Paulo, SP - Brasil. 
BENfCIO, M.H.DA. et al. Estado nutricional dos pré-escolares ingressantes nos Centros de Educação e Alimentação do Pré-Escolar. Rev.Saúde públ., S. Paulo, 15(supl.): 33-9, 1981.

\section{MATERIAL E MÉTODOS}

A população de estudo consistiu da totalidade dos pré-escolares inscritos nos CEAPEs de 4 municípios do Estado de São Paulo (Leme, Indaiatuba, Campinas e Sumaré) em 1978 e 1979.

O estado nutricional dos pré-escolares foi avaliado por intermédio da medida do peso e da altura. Tais medidas foram realizadas utilizando-se técnica recomendada por Jelliffe * sobre avaliação do estado nutricional na comunidade, adaptada pelo Departamento de Nutrição da Faculdade de Saúde Pública da Universidade de São Paulo. Cada medida foi tomada duplamente por dois antropometristas treinados e os resultados foram conferidos, sendo refeitas as medidas com diferenças superiores a $100 \mathrm{~g}$ para o peso e $0,5 \mathrm{~cm}$ para a estatura. Neste caso o exame antropométrico era repetido até que as diferenças entre os examinadores se situassem dentro dos limites permitidos.

A tabela de referência utilizada como padrão de peso e altura foi a de Santo André classe IV 5. Os dados obtidos foram comparados com os valores médios da classe IV e calculadas as adequações do peso para a idade, peso para a altura e altura para a idade. A adequação do peso para a idade, ao desconsiderar a estatura da criança, expressa déficits relacionados não somente com os processos atuais de dpc como também com aqueles ocorridos no passado. Segundo a classificação de Gomez ${ }^{2}$, adequaçōes situadas entre 90 e $75 \%$ caracterizam o primeiro grau de desnutrição $\left(D_{I}\right)$, de 75 a $60 \%$ o segundo grau $\left(D_{\mathrm{II}}\right)$ e inferiores a $60 \%$ o terceiro grau $\left(D_{I I I}\right)$.

A adequação peso/altura reflete processos atuais de dpc sendo aceito que niveis inferiores a $90 \%$ caracterizam a desnutrição atual 1.

A adequação estatura/idade é influenciada exclusivamente pela desnutrição ocorrida no passado. Niveis de adequação inferiores a $92 \%$ caracterizam comprometimento do crescimento em estatura ${ }^{1 .}$
Os resultados obtidos a partir da utilização destes instrumentos classificatórios foram descritos segundo a faixa etária dos pré-escolares e testadas as associações entre aqueles resultados e a idade, por intermédio do teste de $\mathrm{x}^{2}$ adotando-se um nível crítico de $5 \%$ para o risco de falsa rejeição da hipótese nula.

Os resultados encontrados foram comparados com os descritos em populações de baixa renda do Municipio de São Paulo e de núcleos rurais do Vale do Ribeira no Estado de São Paulo.

\section{RESULTADOS}

\section{$O$ Estado Nutricional dos Ingressantes nos CEAPEs.}

Observa-se na Tabela 1 que as médias de peso encontradas no grupo de ingressantes nos CEAPEs são sistematicamente inferiores às do padrão de referência para a população brasileira. $O$ diferencial, entre as duas populações aumenta com a idade sendo igual a $1,2 \mathrm{k}$ aos 2 anos e cerca de $2,3 \mathrm{~kg}$ aos 6 anos.

A média de altura dos pré-escolares ingressantes nos CEAPEs também é sistematicamente inferior a do padrão brasileiro ainda que não haja aumento da diferença com o progredir da idade (Tabela 2 ).

Segundo o critério de Gomez a prevalência de desnutridos nesta população é igual a $46,8 \%$, com graus moderados e graves em $5,8 \%$ dos casos (Tabela 3). Desnutrição atual segundo o critério de adequação do peso para a altura aparece em $19,9 \%$ das crianças e déficit de estatura ocorre em $12,6 \%$ delas (Tabela 4).

O critério de Gomez por desconsiderar a estatura engloba casos de desnutrição atual e pregressa. Assim segundo este critério a prevalência de desnutrição mostra tendência a aumento com o avançar da idade, não havendo no entanto associação estatisticamente significante entre a prevalência de dpc e o fator idade, 
BENfCIO, M.H.D'A. et al. Estado nutricional dos pré-escolares ingressantes nos Centros de Educação e Alimentação do Pré-Escolar. Rev.Saúde públ., S. Paulo, 15(supl.): 33-9, 1981.

T A B E L A 1

Média de peso de pré-escolares ingressantes nos CEAPEs.

\begin{tabular}{cccc}
\hline & \multicolumn{3}{c}{ Pré-escolares } \\
\cline { 2 - 4 } $\begin{array}{c}\text { Idade } \\
\text { (anos) }\end{array}$ & \multicolumn{2}{c}{$\begin{array}{c}\text { Ingressantes nos CEAPEs * } \\
(\mathrm{N}=612)\end{array}$} & Norma \\
\cline { 2 - 4 } & $\begin{array}{c}\text { Média } \\
\text { (kilos) }\end{array}$ & (D.P.) & $\begin{array}{c}\text { Média } \\
\text { (kilos) }\end{array}$ \\
\hline 2 & 12,58 & 0.21 & 13,83 \\
3 & 14,67 & 0,22 & 15,69 \\
4 & 16,46 & 0,23 & 17,58 \\
5 & 18,13 & 0,25 & 19,71 \\
6 & 19,72 & 0,26 & 22,04 \\
\hline * Dados referentes aos CEAPEs de Sumaré, Indaiatuba, Leme e Capivari. \\
Santo André, Classe IV.
\end{tabular}

T A B E L A 2

Média de altura de pré-escolares ingressantes nos CEAPEs.

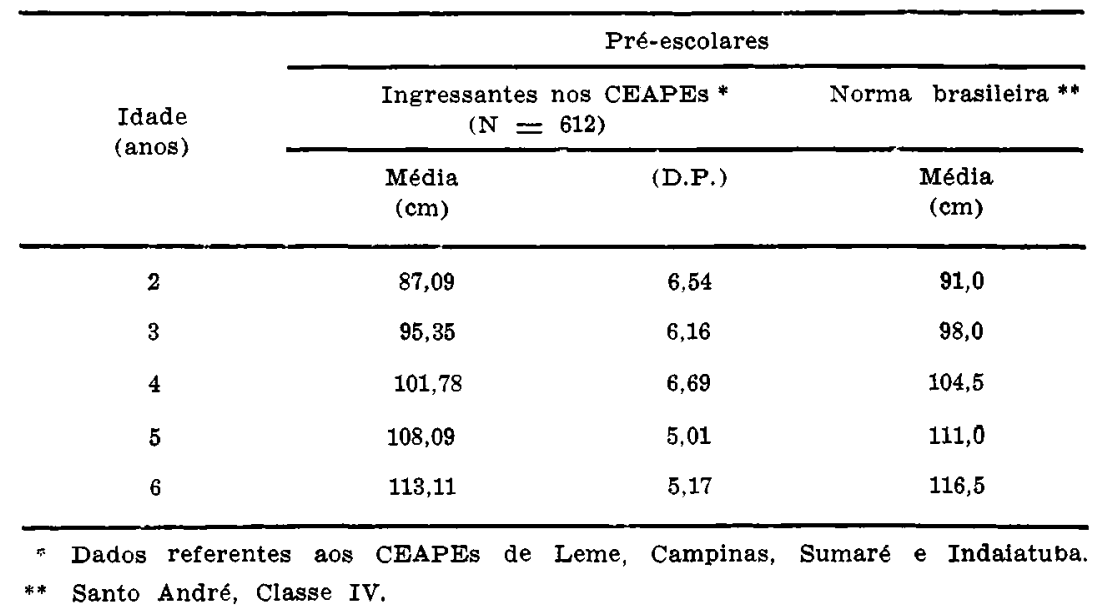

Na tabela 4 verifica-se que é a desnutrição atual a que tende a mostrar percentuais mais elevados nas faixas etárias superiores.

Os déficits de estatura mostram um comportamento oscilante com um aumento do percentual de crianças consideradas inadequadas até a faixa dos 3 anos para a partir de então haver nítida tendência ao declínio destes percentuais.

A análise estatística por intermédio do teste de $x^{2}$ mostra uma associação significativa entre a idade da criança e a desnutrição atual como também entre o fator idade e a desnutrição pregressa. 
BENfCIO, M.H.D'A. et al. Estado nutricional dos pré-escolares ingressantes nos Centros de Educação e Alimentação do Pré-Escolar. Rev.Saúde públ., S. Paulo, 15(supl.): 33-9, 1981.

TA B E L A 3

Pré-escolares ingressantes nos CEAPEs, segundo a idade e a classificação de Gomez. Estado de São Paulo, 1977 e 1978.

\begin{tabular}{|c|c|c|c|c|c|c|}
\hline & \multirow{2}{*}{$\begin{array}{l}\text { Idade } \\
\text { (anos) }\end{array}$} & \multirow{2}{*}{$\begin{array}{l}\text { No de crianças } \\
\text { examinadas }\end{array}$} & \multicolumn{4}{|c|}{ Classificação de Gomez (\%) } \\
\hline & & & $\mathrm{N}$ & $\mathrm{D}_{\mathrm{I}}$ & $D_{T I}$ & $D_{\text {II }}$ \\
\hline & $21-3$ & 49 & 55,1 & 38,8 & 6,1 & 0,0 \\
\hline & $3-4$ & 80 & $\mathbf{5 7 , 6}$ & 36,2 & 6,2 & 0,0 \\
\hline & $4-5$ & 106 & 58,5 & 34,9 & 5,7 & 0,9 \\
\hline & $5-6$ & 201 & 51,9 & 44,2 & 3,9 & 0,0 \\
\hline & $61-7$ & 176 & 49,0 & 43,7 & 7,3 & 0,0 \\
\hline Total & & 612 & 53,2 & 41,0 & $\mathbf{5 , 7}$ & 0,1 \\
\hline
\end{tabular}

T A B E L A 4

Pré-escolares ingressantes nos CEAPEs, segundo a idade, e as adequações peso/altura e altura/idade. Estado de São Paulo, 1977 e 1978.

\begin{tabular}{cccc}
\hline $\begin{array}{c}\text { Idade } \\
\text { (anos) }\end{array}$ & $\begin{array}{c}\text { No de crianças } \\
\text { examinadas }\end{array}$ & $\begin{array}{c}\text { Adequação } \\
\text { peso/altura } \\
<90 \%\end{array}$ & $\begin{array}{c}\text { Adequação } \\
\text { altura/idade } \\
<92 \%\end{array}$ \\
\hline $2 \mid-3$ & 49 & $\%$ & $\%$ \\
$3 \mid-4$ & 80 & 16,2 & 12,3 \\
$4 \mid-5$ & 106 & 13,6 & 21,2 \\
$5 \mid-6$ & 201 & 13,1 & 16,0 \\
& 176 & 23,6 & 9,5 \\
\hline Total & 176 & 24,3 & 10,2 \\
\hline
\end{tabular}

Dada a disponibilidade de dois estudos epidemiológicos realizados no Estado de São Paulo quantificando a prevalência da desnutrição em menores de 5 anos em populaçōes desprivilegiadas economicamente torna-se oportuna a comparação de nossos dados com os achados daqueles autores.

O Estado Nutricional dos Ingressantes Comparado ao de Outros Pré-Escolares de Baixa Renda de São Paulo.

Chamando a atenção para o fato de que na Tabela 5 foram incluidos apenas os ingressantes menores de 5 anos, pode-se observar que a prevalência global de desnutrição segundo o critério de Gomez nestes pré-escolares, situa-se entre a encontrada por Monteiro " em núcleos rurais do Vale do Ribeira e aquela descrita por Sigulem ${ }^{9}$ na população de baixa renda do município de São Paulo. Se considerarmos apenas os casos moderados e graves de dpe segundo Gomez (a soma dos desnutridos de II e III graus) a situação se mantém na medida em que o Vale do Ribeira mostra uma prevalência igual a $11,5 \%$, os ingressantes $6,4 \%$ e a baixa renda de São Paulo $5 \%$. 
BENfCIO, M.H.D.A. et al. Estado nutricional dos pré-escolares ingressantes nos Centros de Educação e Alimentaçăo do Pré-Escolar. Rev.Saúde públ., S. Paulo, 15(supl.): 33-9, 1981.

T A B E L A 5

Ingressantes nos CEAPEs e crianças menores de 5 anos de baixa renda do Estado de São Paulo, segundo a) classificação de Gomez, 1975 - 1978.

\begin{tabular}{lccccc}
\hline & & \multicolumn{4}{c}{ Classificação de Gomez (\%) } \\
Crianças & Total & $\mathrm{N}$ & $\mathrm{D}_{\mathrm{I}}$ & $\mathrm{D}_{\mathrm{II}}$ & $\mathrm{D}_{\mathrm{III}}$ \\
\hline $\begin{array}{l}\text { Ingressantes * } \\
\text { nos CEAPEs }\end{array}$ & 265 & 57,4 & 36,2 & 6,0 & 0,4 \\
Vale do Ribeira (a) & 913 & 50,5 & 38,0 & 10,8 & 0,7 \\
$\begin{array}{l}\text { Município de } \\
\text { São Paulo (b) }\end{array}$ & 200 & 60,5 & 34,5 & 4,5 & 0,5 \\
\hline
\end{tabular}

* Nesta tabela foram incluidos somente os ingressantes nos CEAPEs menores de 5 anos.

Fontes:

a) Monteiro, C.A. - A Epidemiologia da Desnutrição Proteico-Calórica em Núcleos Rurais do Vale do Ribeira (Tese).

b) Sigulem, D.M. - Contribuição ao Estudo da Desnlitrição Energético-Proteica em Crianças de 6 a 60 meses no município de São Paulo (Tese).

No tocante à desnutrição atual, segundo aqueles autores a prevalência mais elevada se encontra na baixa renda do município de São Paulo com nível igual a $20 \%$, contra $13,6 \%$ e $11,6 \%$ respectivamente nos Ceapenses e no Vale do Ribeira.

Já o comprometimento estatural indicativo de processos antigos de dpc ocorre mais freqüentemente no Vale do Ribeira, onde o percentual de déficit de adequação inferior a $90 \%$ é igual a $16,7 \%$, apresentando a baixa renda do município de São Paulo $e$ os ingressantes nos CEAPEs níveis menos elevados, iguais a $9,5 \%$ e $4,1 \%$ respectivamente.

\section{DISCUSSäO}

O objetivo deste trabalho foi o estudo da prevalência da dpc nos pré-escolares ingressantes nos CEAPEs. Os resultados indicam a existência de um retardo no crescimento físico expresso pelos diferenciais encontrados entre as médias de peso e estatura de Ceapenses e as médias da população brasileira padrão. No entanto o comportamento destes parâmetros segundo a faixa etária mostrou-se desigual - a diferença de peso entre as duas populações aumentou com a idade enquanto o diferencial de estatura se manteve inalterado.

Instrumentos que possibilitam quantificar a magnitude da dpc nesta população indicaram prevalência não elevada de formas moderadas e graves do processo.

Considerando-se a classificação de Gomez a qual reflete a somatória de formas atuais e pregressas de dpc observa-se ausência de associação entre dpc e idade. Já os déficits de estatura indicativos de processos passados de dpc sofrem influência da faixa etária sendo mais freqüentes nas crianças com idade inferior aos 4 anos. A partir de então parece ocorrer todo um esforço no sentido de privilegiar o crescimento em estatura como uma tentativa de minimizar os déficits decorrentes de retardo ocorrido em idades mais precoces, época em que o efeito da desnutrição sobre o crescimento estatural se daria de forma mais contundente.

Os dados sugerem que este esforço no sentido de privilegiar o crescimento em estatura a partir dos 4 anos, contribua para que haja diminuição da relação peso/altura acima dos 4 anos. 
BE.NfCIO, M.H.D'A. et al. Estado nutricional dos pré-escolares ingressantes nos Centros de Educação e Alimentaçăo do Pré-Escolar. Rev. Saude públ., S. Paulo, 15(supl.): 33-9, 1981.

A comparação entre estes achados e os encontrados em 2 populaçōes bastante desfavorecidas economicamente do Estado de São Paulo situou a prevalência de dpc dos ingressantes nos CEAPEs em posição intermediária entre os núcleos rurais do Vale do Ribeira e a baixa renda do municipio de São Paulo. Segundo Monteiro ${ }^{6}$ no Vale do Ribeira os agravos nutricionais acometem as crianças de maneira lenta e constante fazendo com que os organismos se "adaptem" à situação retardando o crescimento em estatura a fim de adequá-los aos insumos existentes mantendo assim uma certa condição de homeostase. Na baixa renda do município de São Paulo ocorrem mais freqüentemente agravos nutricionais intensos mas passageiros, decorrentes da associação infecção-desnutrição, que comprometem a adequação peso/altura mas que não chegam a interferir de maneira importante no crescimento em estatura.

A posição intermediária do estado nutricional dos ingressantes nos CEAPEs em relação ao Vale do Ribeira e a baixa renda do município de São Paulo deve-se possivelmente a não existência dos graves problemas de saneamento que em São Paulo dão origem ao elevado percentual de processos agudos de dpc e possivelmente a menor magnitude do déficit de consumo alimentar em relação aos núcleos rurais do Vale do Ribeira estudados.

\section{CONCLUSOES}

- Os ingressantes nos CEAPEs apresentam retardo do crescimento pondero-estatural, sendo que os diferenciais de crescimento em relação ao padrão brasileiro se mantém inalterados quanto à estatura $\mathrm{e}$ aumentam com o avançar da idade no que se refere ao peso.

- Segundo o critério de Gomez predominam formas leves e moderadas de dpc nestes pré-escolares.

- Os déficits de estatura, indicativos de desnutrição pregressa, tendem a diminuir enquanto a adequação peso/altura associada à desnutrição atual mostra tendência a piora com o avançar da idade.

- A magnitude da desnutrição nos ingressantes dos CEAPEs situa-se em posição intermediária entre as descritas em núcleos rurais do Vale do Ribeira e na baixa renda do município de São Paulo.

BENfCIO, M. H. D'A. Et al. [Nutritional status of preschoolers entering the CEAPEs].

Rev. Saúde públ., S. Paulo, 15(suppl.):33-9, 1981.

ABSTRACT: The nutritional status of CEAPE entrees is described by means of anthropometric measurements. Prevalence of PEM encountered was $46.8 \%$ for moderate degrees and $5.8 \%$ for severe ones. This situation is intermediate between two other economically handicaped populations described in the State of S. Paulo, Brazil.

UNITERMS: Child, preschool. Nutritional status. CEAPE. 
BENfCIO, M.H.D'A. et al. Estado nutricional dos pré-escolares ingressantes nos Centros de Educação e Alimentação do Pré-Escolar. Rev. Saúde públ., S. Paulo 15(supl.): 33-9, 1981.

\section{REFERENCIAS BIBLIOGRAFICAS}

1. BATISTA, M. Prevaléncia e estágios da desnutriøão proteico calórica em criangas da cidade de São Paulo. São Paulo, 1976. [Tese de Doutoramento - Faculdade de Saúde Pública da USP]

2. GANDRA, Y.R. Asistencia alimentaria por medio de centros de educación y alimentación del pre-escolar. Bol. Ofic. sanit. panamer., 74:302-14, 1973.

3. GOMEz, F. Desnutrición. Bol. méd. Hosp. Inf., Mexico, 3:543-51, 1946.

4. JELLIFFE, D. Evaluación del estado de nutricion de la comunidad: con especial referencia a las encuestas en las regions en desarollo. Ginebra, 1968. (OMS Série de Monografias, 53).

5. MARQUES, R.M.; BERQUO, E.; YUNES, J. \& MARCONDES, E. Crecimiento de niños brasileños: peso y altura en relación con la edad y el sexo y la influencia de factores socioeconomicos. Washington, D.C., Organizacion Panamericana de la Salud, 1975. (OPAS - Publ. cient., 309).

6 MONTEIRO, C.A. A epidemiologia da desnutrição proteico-calórica em núcleos rurais do Vale do Ribeira. São Paulo, 1977. [Dissertação de Mestrado - Faculdade de Medicina da USP]

7. RELATório de atividades do Grupo de Pesquisas de Ciências Sociais em Nutrição da FINEP/INAN/IBGE, 6?. Rio de Janeiro, 1979.

8. RELATORIO de atividades do Grupo de Pesquisas de Ciências Sociais em Nutrị̧ão da FINEP/INAN/IBGE, 8*. Rio Janeiro, 1980.

9. SIGULEM, D.M. Contribuicão ao estudo dı da desnutrição energético-proteica em criancas de 6 a 60 meses no municipio de São Paulo. São Paulo, 1980. [Dissertação de Mestrado - Escola Paulista de Medicina]

10. VIEIRA, J.L.T. $O$ impacto da renda no estado nutricional das familias paulistanas. São Paulo, 1976. [Dissertação de Mestrado - Instituto de Pesquisas Econômicas da USP]

Recebido para publicasăo em 10/0\%/1981

Aprovado para publicasão em 1\%/11/1981 